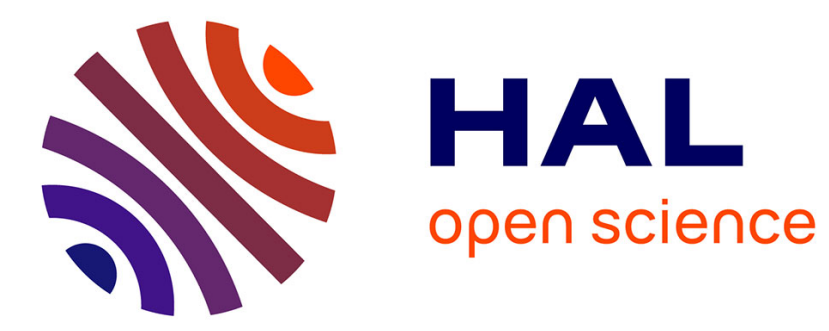

\title{
Psychopathologie et expériences exceptionnelles : une revue de la littérature
}

\author{
Renaud Evrard
}

\section{To cite this version:}

Renaud Evrard. Psychopathologie et expériences exceptionnelles : une revue de la littérature. L'Évolution Psychiatrique, 2013, 78 (1), pp.155-176. 10.1016/j.evopsy.2013.01.006 halshs-02137222

\section{HAL Id: halshs-02137222 \\ https://shs.hal.science/halshs-02137222}

Submitted on 22 May 2019

HAL is a multi-disciplinary open access archive for the deposit and dissemination of scientific research documents, whether they are published or not. The documents may come from teaching and research institutions in France or abroad, or from public or private research centers.
L'archive ouverte pluridisciplinaire HAL, est destinée au dépôt et à la diffusion de documents scientifiques de niveau recherche, publiés ou non, émanant des établissements d'enseignement et de recherche français ou étrangers, des laboratoires publics ou privés. 
Evrard, R. (2013). Psychopathologie et expériences exceptionnelles : une revue de la littérature. L'Evolution Psychiatrique, 78(1), 155-176.

\section{Introduction}

Plusieurs chercheurs dans le champ de la santé mentale ont perçu une interface possible entre clinique et parapsychologie, l'étude scientifique des phénomènes dits paranormaux. Celle-ci reçut des appellations variées : «métapsychiatrie » [1], «parapsychiatrie » [2], «parapsychologie clinique »[3], «parapsychoanalyse »[4], etc. Ce champ intermédiaire était au carrefour de plusieurs écoles de psychologie, avant de s'autonomiser pour devenir une spécialité à part entière : la « clinique des expériences exceptionnelles », dont la «psychologie anomalistique » est le pendant dans les sphères de la recherche et de l'enseignement. Cette revue de littérature a pour but d'introduire à ces domaines, dont les dernières décennies ont été riches d'avancées, principalement en Angleterre et en Allemagne. L'accent sera mis sur certaines des interactions entre la psychopathologie et les expériences exceptionnelles, et non sur les recherches empiriques visant à découvrir la nature ontologique de ces vécus. Cette mise en garde semble nécessaire car le « paranormal » a plus souvent donné lieu à des polémiques infinies qu'à des examens scientifiques dépassionnés [5-6].

\section{Approches du discours parapsychologique}

On constate plusieurs croisements possibles entre psychopathologie et parapsychologie ([7], pp. 89) : l'implication de cliniciens dans l'étude scientifique du paranormal ou sa critique ; la rencontre dans une pratique clinique de manifestations d'allure paranormale (par exemple, [8]) ; la coloration de certains troubles par des thèmes ou des pratiques relevant du champ du paranormal ; l'utilisation de vocabulaire à thème parapsychologique dans la nosographie; la prise de position, dans un 
discours médico-légal ou d'hygiène mentale, sur la parapsychologie; ou encore la concurrence entre les psychiatres et certains guérisseurs s'invitant dans le champ de la santé mentale.

La complexité de ces interactions nous oblige à nous positionner vis-à-vis du discours parapsychologique. Nous nous inscrivons dans l'épistémologie des recherches psychologiques sur le paranormal [5], c'est-à-dire une étude recentrée sur des individus, sur des « expériences » plutôt que sur des «phénomènes », sans chercher à entrer dans un débat sur la preuve desdits phénomènes paranormaux. Cette psychologie du paranormal invite à se repositionner autour de vécus et de croyances, sans préjuger de leur nature réelle ou illusoire, pathologique ou non-pathologique.

Ce positionnement épistémologique s'appuie notamment sur la notion d'expériences exceptionnelles, que nous allons présenter et situer sur le plan psychopathologique.

\section{Définition des expériences exceptionnelles}

Il n'y a aucune définition des «expériences exceptionnelles » qui fasse actuellement consensus. Ce domaine de recherche est en lui-même très jeune, tout du moins dans sa formalisation actuelle. L'expression «expérience exceptionnelle » vient se substituer à un ensemble d'appellations tirées de diverses traditions de pensée et représentations du monde: expériences «paranormales », «transpersonnelles », « spirituelles », «mystiques », «transcendantales », «numineuses », « surnaturelles », « magiques », «Psi », « extrasensorielles », « non-ordinaires »... pour ne citer que quelques-uns des concepts tentant de rendre compte des expériences en question 1 .

Le terme d'expériences exceptionnelles est désormais largement employé dans le monde académique. La psychologue Martina Belz, de l'université de Berne, motive sa préférence pour ce concept par quatre aspects de cette terminologie ([19], pp. 4-5) :

1 Palmer et Braud ([9], p. 32) repèrent encore d'autres termes dans la littérature transpersonnelle : expériences paroxystiques (peak experiences) [10] ; Minerva experiences [11] ; phénomènes extraordinaires [12] ; expériences transcendantales [13] ; expériences extraordinaires [14] ; expériences préternaturelles [15] ; fonctionnement métanormal [16]; wondrous events [17]; et high holy moments [18]. À cette liste confuse, ils ajoutent un nouveau terme 
([9], p. 32): «expériences non-ordinaires et transcendantes » (nonordinary and transcendent experiences, ou NOTE) terme qui, selon eux, rend compte de la relative rareté et de la non-familiarité de ces expériences, et aussi de leur tendance à aller au-delà de la compréhension conventionnelle de soi-même et du monde, et enfin de leur propension à déclencher des transformations qui permettent de transcender ce qu'on était.

A elle reste neutre du point de vue de la vision du monde ;

A elle évite l'amalgame avec un trouble psychique et ne suggère aucun diagnostic ;

A elle prend en compte la qualité particulière du vécu ;

A et elle ne répond pas par elle-même à la question de la factualité.

Cette définition se conforme donc à certaines exigences de travail. Ce ne sont donc pas des contraintes de langage qui sont à la base de l'élection de ce terme, mais des orientations du dispositif thérapeutique. Par exemple, le vocable Aussergewöhnliche Erfahrungen a été intégré dans les activités de l'Institut pour les Zones frontières de la Psychologie et l'Hygiène mentale (IGPP), fondé à Freiburg-in-Breisgau par le Dr Hans Bender en 1950, tant pour le département de consultation et d'information que dans les recherches sociologiques, neurophysiologiques, etc. Plusieurs travaux universitaires en Allemagne emploient désormais ce mot-clef [20-24].

Au-delà de cette première définition opérationnelle, les travaux allemands s'orientent vers une autre définition des expériences exceptionnelles qui soit moins dépendante des catégories manipulées par les parapsychologues, pour se saisir davantage de l'évaluation subjective du «paranormal » :

«Les expériences exceptionnelles sont des expériences vécues avec une qualité subjective si particulière et qui s'écartent si distinctement des modèles explicatifs de ceux qui les vivent, qu'elles ne sont pas intégrées dans les schémas cognitifs et émotionnels disponibles. » 2

Les expériences exceptionnelles peuvent ainsi être abordées comme un problème concret et contemporain posé à la psychopathologie et à la clinique [25], déclinaison actuelle de la rencontre entre des savoirs psychopathologiques et le point de vue d'un sujet: comment aider un individu croyant faire une expérience subjective hors du commun? Ces expériences jouent également un rôle transversal dans l'histoire de la psychopathologie, ayant eu une influence souvent négligée sur les nosographies actuellement en usage [26]. Leur étude permet d'explorer les marges de la clinique, 
tout en renvoyant constamment à la question de la position du clinicien. Le cœur même de la

2 Belz-Merk M. Beratung und Hilfe für Menschen mit Außergewöhnlichen Erfahrungen. Rapport final non publié, Université Albert-Ludwigs de Freiburg ; 2002, p. 12-13.

clinique est de s'orienter vers un autre discours et une autre écoute que ceux du chercheur ou de l'homme du commun. Les hallucinations, les délires, ou, via une autre formulation du problème, les expériences exceptionnelles, engendrent une cascade de provocations à l'égard d'un savoir psychopathologique qui, à la fois, les domine et reste travaillé par eux [27].

\section{Exceptionnel, anormal et anomal}

Le terme d'expériences exceptionnelles ne fait pas l'unanimité dans les pays anglo-saxons car il est supplanté par celui d'anomalous experiences (expériences anomales), qui doit son succès à l'important ouvrage Varieties of Anomalous Experiences, publié par l'American Psychological Association [28]. Cette somme académique synthétise sur douze chapitres toute la recherche sur les expériences de hors corps, de mort imminente, les rêves lucides, les expériences d'enlèvement par des extra-terrestres, etc. Mais le terme d' «expériences anomales »n'accompagne pas de pratiques cliniques, et son usage se restreint à des unités de recherche universitaires. On le retrouve dans le nom de groupes de recherche en psychologie ou sociologie en Europe, principalement dans plus d'une douzaine d'universités au Royaume-Uni.

L'adjectif «anomal » est très peu usité en français, tombé en désuétude au XIXe siècle. L'usage l'a couplé avec l'adjectif «anormal », ce qui pose problème ici. Dans son Vocabulaire technique et critique de la philosophie, Lalande [29] expose cette confusion d'étymologie qui a aidé à un rapprochement entre les mots «anomalie» et «anormal ». Anomalie vient du grec anomalia qui signifie inégalité, aspérité ; omalos désigne en grec ce qui est uni, égal, lisse, en sorte que anomalie c'est étymologiquement an-omalos, ce qui est inégal, rugueux, irrégulier, au sens qu'on donne à ces mots en parlant d'un terrain. Or, on s'est souvent mépris sur l'étymologie du terme anomalie en le 
dérivant, non pas de omalos, mais de nomos qui signifie loi, selon la composition a-nomos.

Georges Canguilhem rappelle que cette erreur d'étymologie se retrouve dans des dictionnaires de médecine ([30], p. 81). Cette collusion participe des définitions confuses de la normalité et de la pathologie. Le terme d'anomalie a un sens d'insolite, d'inaccoutumé. Ainsi, en toute rigueur sémantique, anomalie désigne un fait qu'il faut expliquer, c'est un terme descriptif, alors que anormal implique la référence à une valeur, c'est un terme appréciatif, normatif. Mais les bons procédés grammaticaux ont entraîné une collusion des sens respectifs d'anomalie et d'anormal. Cette collusion ne semble pas levée aujourd'hui ce qui fait que, dans la langue de Molière, parler d'expérience anomale (anomalous experience) entraîne une trop grande confusion avec l'expérience anormale (abnormal experience).

Or, la différence d'appellation a un impact important quant à la question du diagnostic différentiel. Comme le rappelle Canguilhem ([30], p. 85), «l'anomal ce n'est pas le pathologique », car le pathologique implique pathos, sentiment direct et concret de souffrance et d'impuissance, sentiment de vie contrariée, «mais le pathologique c'est bien l'anormal ». L'étude des anomalous experiences s'inscrit davantage du côté de la psychologie différentielle, c'est-à-dire les études des individual differences au pays de Shakespeare. En effet, l'anomal n'est pas situé directement du côté du pathologique et de la clinique, c'est un point de vue plus philosophique que Canguilhem rapproche du principe leibnizien des indiscernables : «L'anomalie c'est le fait de variation individuelle qui empêche deux êtres de pouvoir se substituer l'un à l'autre de façon complète » ([30], p. 85). Cela permet d'extraire l'adjectif anomal d'une référence à une norme ou à une loi, pour le ramener au statut d'une exception par rapport à une règle (voir Tableau 1).

\section{Tableau 1}

Toutefois, dans l'archéologie effectuée par Foucault [31], les anomalies et l'exceptionnalité sont présentées comme les objets privilégiés de la psychiatrie. Ainsi, de la fin du XVIIIe siècle jusqu'au début du XIXe, les équivoques sont nombreuses autour de la figure du «monstre » qui reste 
« l'exceptionnel », puis d'un glissement vers «l'individu à corriger », c'est-à-dire l'anormal, le

« monstre banalisé » 3 ([31], p. 53). À partir du milieu du XIXe siècle, la psychiatrie se définirait

3 Robert Castel ([32], p. 132) propose le néologisme d'anomaliques pour désigner ces déviants ordinaires peuplant les «populations à risque ».

même comme «technologie de l'anomalie » ([31], p. 151). Chez Foucault, personnage de l'anormal et domaine des anomalies sont confondus. Cette perspective rend difficile l'expansion d'une « psychologie anomalistique » qui intègre les hypothèses psychopathologiques sans s'y réduire (par exemple, avec les hypothèses de perméabilité psychique, schizotypie positive, inclination à l'imaginaire et transliminalité ; voir [33]).

Au final, l'appellation « expériences exceptionnelles » nous a semblé celle qui se rapprochait le plus du sens philosophique et étymologique de l'anomal, sans évoquer la collusion habituelle avec l'anormal.

\section{Données épidémiologiques}

Un aspect qui justifie la prise en compte des expériences exceptionnelles est leur présence importante dans toutes les couches de la population et dans tous les pays [34-35]. La sociologie a fourni de nombreuses données qui peuvent être utilisées pour une épidémiologie de ces expériences. Cependant, il faut pouvoir analyser la façon dont sont récupérées ces données. Les chiffres varient en effet selon que l'on mesure les croyances ou les expériences (quand on prend soin de distinguer les deux, voir plus loin), selon la façon dont sont formulées les questions, selon les catégories d'expériences définies par les sondeurs, selon la population à laquelle le sondage est administré, etc. Cela rend toute interprétation précise difficile. Nous nous contenterons d'une analyse globale se concentrant sur les traits dominants qui ressortent de ces données.

Bien qu'on les appelle «exceptionnelles » ou «paranormales », ces expériences sont vécues par une population si large qu'elle en dépasse la «norme statistique ». Dans l'étude de Schmied-Knittel 
\& Schetsche [36] réalisée en Allemagne, $73 \%$, soit environ trois quart des sondés, disent avoir vécu une expérience paranormale subjective au moins une fois dans leur vie. Cependant, ce sondage inclut le déjà-vu en tant qu'expérience paranormale, qui obtient le plus haut score de représentativité avec 49,5\%. Or, comme le déjà-vu a pu être normalisé [37], et même s'il peut constituer la base d'une expérience exceptionnelle, il nous semble trop ambigu d'intégrer un tel phénomène dans cette liste. Les sondés ne doivent pas avoir autant de scrupules à affirmer avoir vécu une expérience qui ressemble au déjà-vu, par rapport à d'autres expériences comme l'observation d'OVNI (qui ne touche que 2,4\% des sondés).

Un chiffre global moins biaisé, se basant sur des données de nombreux pays, serait de dire qu'entre $30 \%$ et $50 \%$ des personnes reconnaissent avoir vécu une expérience exceptionnelle au moins une fois dans leur vie ([19], p. 16). Ce chiffre important a fait dire à des auteurs que « le paranormal est normal » [35]; ou encore qu'il serait en fait plus intéressant de comparer des personnes ayant une unique expérience exceptionnelle à des personnes ayant plusieurs expériences, ce que nous ferons dans notre recherche, plutôt que la comparaison classique entre ceux qui relatent et ceux qui ne relatent pas d'expériences ([38], p. 260).

La croyance aux phénomènes paranormaux serait plus répandue : entre $60 \%$ et $75 \%$ des personnes croient au moins à un phénomène paranormal [39].

La prévalence élevée de ces expériences aboutit nécessairement à ce qu'elles soient discutées dans le cadre psychothérapeutique. Dans une étude ciblée aux Pays-Bas [40] sur 640 professionnels de santé sondés, 129 ont retourné les questionnaires, dont environ 1 sur 2 affirmant avoir été contacté au moins une fois par une personne vivant des expériences exceptionnelles. Environ 4 de ces professionnels sur 5 disaient ne pas avoir de connaissances suffisantes pour traiter ce cas. Une estimation prenant en compte le nombre de contacts et le nombre de non-répondants évalue que chaque professionnel de santé est amené au moins une fois dans sa carrière à être abordé par une personne en détresse par rapport à son expérience exceptionnelle ([41], p. 43).

Les expériences ne se répartissent pas équitablement dans la population, même s’il faut souligner ce 
fait (étayé entre autres par Sannwald, [42]) : il n’y a pas un seul groupe d'individus spécifiques qui vivrait ces expériences, mais tous les types de populations. Les données empiriques invalident effectivement l'hypothèse de la «marginalité »[43] attribuant toutes ces expériences à une population en marge. On a pu remarquer que les expériences étaient davantage rapportées par des femmes que par des hommes; et qu'elles variaient en fonction de l'âge : des populations âgées rapportaient plus d'expériences en lien avec des apparitions, des possessions ou la mort; des populations de jeunes témoignaient plus d'expériences de déjà-vu, de coïncidence significative et d'observation d'OVNI [44]. Plusieurs hypothèses tentent de rendre compte de ce type de différences dans les données [39].

Finalement, ni la rareté ni la spécificité sociopsychologique ne sont des composantes essentielles de l'exceptionnalité caractérisant ces expériences. Cela étant, la présence importante de celles-ci justifie que les sciences humaines leur portent une grande attention. En allant même plus loin, Ross et Joshi affirment que :

«Les expériences paranormales sont si communes dans la population générale qu'aucune théorie de la psychologie normale ou de la psychopathologie ne peut être complète si elle ne les prend pas en compte. » ([45], p. 360)

Commentant ce propos, Hufford ([46], p. 362) ajoutera que, dans les faits, la théorie psychiatrique conventionnelle ne prend pas en compte ces expériences. L'affirmation de Ross et Joshi est donc éminemment provocante : non seulement les théories psychiatriques ne seraient actuellement pas complètes, mais elles seraient mises en défaut par ces données empiriques qu'elles n'ont pas encore intégrées.

\section{Des hallucinations dans la population générale ?}

Des progrès sont néanmoins faits sur ce plan car les recherches récentes sur les hallucinations dans la population générale commencent à accueillir ces données [47, 27]. Ainsi, pour prendre un 
exemple précis, les hallucinations autour du deuil, comme la vision ou l'audition du proche défunt, la sensation réaliste de sa présence ou de son contact, font parties des expériences désormais reconnues dans la littérature sur le chagrin et le deuil sans être systématiquement associées à la psychopathologie (par exemple, [48]). De nombreuses enquêtes épidémiologiques ont pu interroger des populations du monde entier sur leur «impression d'avoir réellement été en contact avec une personne décédée » : il ressort en moyenne qu'un quart des européens sondés admettent un tel contact direct [49], chiffre qui grimpe à un sur deux pour les veufs et veuves [34]. Dans des pays où ces expériences sont moins stigmatisées, comme le Japon, ces chiffres grimpent encore : à titre d'exemple, $90 \%$ des veuves interrogées par Yamamoto et al. [50] affirmaient avoir un contact avec leur défunt mari.

Ce phénomène de vécu subjectif de contact avec un défunt n'est plus si marginal et commence à être appréhendé au cours des formations de soignants, notamment en soins palliatifs [51]. Des cas publiés dans des revues médicales viennent montrer l'aspect salutaire de ces hallucinations [52]. Le psychanalyste Allouch [53] parle de son côté du phénomène de «vivance » lorsqu'une personne récemment endeuillée croît reconnaître, à partir d'une image faisant signe, la présence réelle de l'être perdu. Il explique ce phénomène par le fait que le deuil est « une des expériences possibles de la perte de la réalité. (...) la réalité ne fait plus paravent au réel » ([53], p. 67), au sens où la réalité est mise à l'épreuve par la mort. Il conçoit le deuil comme «parapsychose » fondée sur une opération inverse de la forclusion: un trou dans le réel qui appelle le symbolique et l'imaginaire. Les possibles phénomènes parapsychotiques seront alors la «quasi-hallucination » de la vivance, l'atteinte à l'image du corps de l'endeuillé, la convocation des éléments symboliques liés au mort, et les «"folies collectives" du type croyance aux fantômes » ([53], p. 333).

Le Maléfan et Lemercier [54] exploitent cette conception lacanienne du deuil pour montrer comment on peut comprendre certaines expériences exceptionnelles autour du ghost (apparition d'un revenant, illusion de présence, hantise, etc.) comme un désordre transitoire provoqué par le trou laissé par le disparu. Ce désordre ne serait pas alors de l'ordre de la psychose (où l'hallucination 
est un retour dans le réel du symbolique forclos), ce qui l'incite à en parler comme une quasihallucination du deuil ou une illusion, conformément à cette différence fondée structuralement et à ce que proposent certains spécialistes de la clinique du deuil ([55], p. 351).

Le constat de Hufford [46] doit donc être nuancé puisque, par des moyens plus ou moins directs, la psychopathologie contemporaine commence à intégrer certaines expériences exceptionnelles avec leurs spécificités. Plus généralement, les expériences exceptionnelles viennent bousculer les modèles de la psychose puisqu'elles suggèrent un continuum entre des hallucinations dans la population générale et des formes graves de maladies mentales telles que la schizophrénie [56].

\section{Science, sociétés et expériences exceptionnelles}

Dans le champ des sciences humaines, l'ethnologie et l'anthropologie se sont penchées à plusieurs reprises sur les rites, les mythes ou les praticiens traditionnels en lien avec des expériences exceptionnelles (cf. par exemple, [57-59]; et depuis 2010 la revue Paranthropology dirigée par Jack Hunter). Ces disciplines mettent en évidence diverses formes d'intégration des expériences exceptionnelles en fonction des cultures, ce qui vient indirectement nous renseigner sur la façon dont les sociétés occidentales abordent ces expériences. Le constat est que nos sociétés ont tendance à davantage s'opposer à leur intégration officielle, développant des formes de « rationalisation » au sens de Max Weber [60] et un monde «désenchanté ». Néanmoins, ces expériences sont omniprésentes dans notre espace culturel, et pas seulement à ses marges [61-62].

Il y a encore d'autres abords empruntant aux sciences humaines et aux sciences de la vie, mais le sujet a également passionné certains chercheurs des sciences de la nature. La parapsychologie ou métapsychique a représenté cette étroite branche scientifique tentant de produire un savoir sur l'authenticité de ces expériences [6, 62]. Ces travaux sont généralement méconnus et marginalisés pour des raisons diverses. Mais il subsiste néanmoins une certaine forme de discours issue de la science même laissant une place à la plausibilité de ces expériences, et qui a des effets importants 
sur les représentations sociales et individuelles de celles-ci. Ce discours pousse à croire qu'il serait naturel que des gens croient aux phénomènes paranormaux et en vivent, si, dans les faits, certains de ces phénomènes existent réellement. Or, même si c'était le cas, cela n'empêcherait pas que ces personnes soient en meilleure ou en moins bonne santé psychologique que d'autres.

Ce discours parapsychologique a toujours rencontré un discours symétrique plaidant le peu de plausibilité de l'authenticité de ces expériences. En dehors des critiques portant sur les expérimentations (par exemple, [63-65, 6]), de nombreuses critiques ont porté directement sur les personnes témoignant d'expériences exceptionnelles [66-68]. À la question : «Pourquoi tant de personnes disent avoir vécu ces expériences?», les trois réponses réductionnistes apportées peuvent se caricaturer sous la forme : «Tous les témoins sont soit des menteurs, soit des fous, soit des imbéciles ». Même si la forme a beaucoup été développée, le fond du modèle sceptique dit composite (au sens où il défend plusieurs hypothèses exclusives en même temps) vise à annuler la valeur en tant que preuves des témoignages d'expériences exceptionnelles.

Cela n'est pas sans effet sur la population des personnes vivant de telles expériences. Ainsi, par des analyses des structures narratives utilisées par les personnes vivant des expériences exceptionnelles, on constate qu'elles anticipent déjà sur les réactions sceptiques des interlocuteurs, par exemple en présentant leur scepticisme antérieur, leurs doutes actuels et en s'en tenant à la factualité de leur vécu [69] ; et en utilisant des formulations suggérant des « chocs existentiels », de l'exceptionnalité ou des transformations «transpersonnelles », c'est-à-dire un ensemble de thématiques floues et supposées saines pour se placer dans un au-delà de la psychopathologie [23,70].

Pour le sociologue Michael Schetsche [71], ces discours symétriques opposant parapsychologues et sceptiques viennent prendre en tenaille les personnes qui vivent des expériences exceptionnelles. Il s'en dégage un discours négatif, généralement partagé par la majorité du corps social et des cliniciens, qui assume ce lien entre «paranormal » et « folie ». Selon lui, c'est cette raison et aucune autre qui fait le lit du mode d'expression contemporain de ces expériences, passant par cette stratégie consistant à assurer à l'auditeur que l'on vit des expériences exceptionnelles sans, 
précisément, être fou.

\section{Développements de la psychologie anomalistique}

Pour sortir des polémiques stériles, des chercheurs sont parvenus à s'entendre pour étudier les expériences exceptionnelles et les croyances au paranormal en commençant par s'appuyer sur des hypothèses psychologiques conventionnelles. Le pari de la psychologie anomalistique est donc que toutes ces expériences pourront être réduites à la psychologie actuelle ou à venir. Des hypothèses sulfureuses comme l'hypothèse parapsychologique (ou psi), si elles restent prises en compte, deviennent moins centrales.

Le premier essai moderne et systématique pour expliquer les expériences exceptionnelles fut The Psychology of Anomalous Experience du psychologue canadien Graham Reed [72]. Cet essai phénoménologique étudie certaines expériences exceptionnelles dans une perspective cognitive et expérientielle.

Le deuxième essai important fut celui de Leonard Zusne et Warren H. Jones [73]. Leur livre Anomalistic Psychology, dont la seconde édition était sous-titrée «Une étude de la pensée magique », s'empêtrait dans un relativisme culturel, où la psychologie devenait une forme d'acculturation au service de la norme statistique et scientifique, prenant pour cibles «ces comportements et ces vécus qui semblent violer les lois de la nature » ([73], p. ix).

À leur suite, d'autres auteurs ont analysé des formes variées d'illusions de la pensée pouvant conduire à croire en certains événements inhabituels. Thomas Gilovich [74] s'intéressa aux croyances à des événements anomaux via leurs déterminants cognitifs (dont la mauvaise perception et la mauvaise interprétation de données aléatoires), motivationnels (comme «voir ce que l'on a envie de voir »), et sociaux (les biais dus aux informations de seconde main). Theodore Schick et Lewis Vaugh [75] discutèrent des nombreuses façons d'ignorer ou de mal représenter une information valable, et de surévaluer dans le même temps des preuves d'apparence douteuse 
reposant sur l'autorité d'une tradition ou de la personne porteuse du message.

Dans ce sillage, la psychologie anomalistique a fait l'objet de nombreuses recherches en psychologie. Les chercheurs les plus marquants furent Blackmore, Wiseman, Morris, Houran, Lange, French, Spanos, Lynn, Loftus, Irwin et Thalbourne. Le Varieties of Anomalous Experiences [28] est un point culminant avec sa publication d'un ensemble de revues de la littérature. Les congrès internationaux ont commencé à intégrer de plus en plus fréquemment des symposiums sur le sujet; de même pour les cours à l'université [76]. Après une décennie, la psychologie anomalistique a ses propres «manuels » [77-78] et même son cours de niveau A dans des classes préparatoires avant l'entrée à l'université au Royaume-Uni [79].

Ainsi, la psychologie anomalistique correspond à une approche scientifique de phénomènes rarement appréhendés, et qui obligent les psychologues à développer des méthodes nouvelles et à faire face à des hypothèses étranges. Cependant, elle n'a pas conduit directement à des applications dans le domaine de la clinique. Par exemple, l'aspect trop descriptif du Varieties ne donnait que peu de pistes pour une confrontation au quotidien avec des personnes vivant des expériences exceptionnelles. De plus, la présentation de la psychologie anomalistique comme une discipline volontairement réductionniste et psychologisante complique la négociation d'un cadre thérapeutique ouvert. Or, dans la pratique clinique, l'objectif n'est pas que le clinicien ait raison, ni qu'il fasse adhérer son patient à la meilleure théorie scientifique connue. Le respect de la réalité psychique et la visée clinique doivent primer.

En somme, la psychologie anomalistique est le pendant de la clinique des expériences exceptionnelles pour ce qui est de la recherche en psychologie, mais les deux domaines ont des bases et des objectifs différents. De nombreuses réflexions sur la clinique des personnes vivant des expériences exceptionnelles ont récemment émergées, en approfondissant cette orientation spécifique [80-82]. 


\section{Problématiques de diagnostic différentiel}

Où en est-on de notre compréhension du rapport des expériences exceptionnelles à la «santé mentale» et à la «folie»? Quelles relations ces vécus entretiennent-ils avec les grandes nosographies internationales, les modèles contemporains de la dissociation, du traumatisme et de l'adolescence? Nous allons passer en revue ces questions pour repérer certains enjeux d'une clinique différentielle de ces expériences.

\section{Expériences exceptionnelles et troubles psychiques}

La question de base est la suivante : quelles sont les zones de chevauchement et de différenciation entre les expériences exceptionnelles et les troubles psychiques?

Dans la littérature, il y a beaucoup de discussion sur les réponses qui ont pu être apportées à cette question. Berenbaum, Kerns et Raghavan ([83], p. 32) disent qu'il n'y a pas suffisamment de données empiriques pour soutenir une association stricte entre les différentes expériences exceptionnelles et la psychopathologie. Cela va dépendre des spécificités de l'expérience mais aussi des critères spécifiques de la psychopathologie. Par exemple, Shafer [84] et Windholz et Diamant [85] n'ont pas pu trouvé de liens entre les croyances au paranormal et la dépression (voir plus loin la distinction entre croyances au paranormal et expériences exceptionnelles).

Non seulement il n'y aurait pas d'association stricte, mais les expériences exceptionnelles contribueraient parfois à la psychopathologie et aussi parfois à une meilleure santé mentale. C'est ce que montrent Reinsel [86] avec des personnes pratiquant la médiumnité, McCreery et Claridge [87] avec des individus ayant des sorties du corps, Jackson [88] avec des personnes ayant des expériences spirituelles intenses, Goulding [89-90] avec des personnalités schizotypiques, ou encore le psychologue clinicien John Schumaker [91] avec des personnes développant à la fois des croyances au paranormal et une meilleure santé mentale telle que mesurée par des questionnaires. 
Ce dernier, qui se présente pourtant comme un «incroyant absolu » au paranormal, a même réalisé une analyse des croyances et expériences paranormales en termes de «bénéfices-risques », d'où il ressort qu'elles seraient des bons moyens de faire face aux aspects les plus durs de la réalité (sur le paranormal comme solution psychodynamique, voir [92]).

C'est là un des premiers paradoxes qui vient d'une rencontre entre certaines expériences et un type d'épistémologie psychopathologique. Par conséquent, toute analyse de ces données implique de bien identifier à la fois de quelle expérience il est question, mais aussi sur quels repères psychopathologiques se fait le diagnostic différentiel.

Les recherches recensées ci-dessus s'entendent au moins sur un point: les expériences exceptionnelles entretiennent des rapports complexes avec la psychopathologie. Berenbaum et al. ([83], p. 32-34) distinguent quatre façons de penser ces rapports :

(a) les deux se chevauchent;

(b) l'expérience contribue à la psychopathologie ;

(c) la psychopathologie contribue à l'expérience exceptionnelle ; et

(d) il y a des « variables tiers » qui contribuent à la fois à l'expérience exceptionnelle et à la psychopathologie.

10.1 Chevauchement complet entre expériences exceptionnelles et psychopathologie

Un premier constat général peut être fait: les données épidémiologiques sur la prévalence de troubles psychiques dans la population générale donnent des chiffres inférieurs à la prévalence d'expériences exceptionnelles. Par exemple, des données récentes en Allemagne montrent une prévalence de troubles psychiques (tous diagnostics confondus) à un moment $t$ (2004) de $31 \%$ de la population générale; et au cours de la vie de $43 \%$ [93]. Connaissant la prévalence élevée des expériences exceptionnelles, il est donc improbable qu'il y ait un chevauchement absolu (hypothèse (a)) entre expériences exceptionnelles et troubles psychiques à moins de considérer des hypothèses 
supplémentaires : par exemple, qu'une grande partie des expériences exceptionnelles n'auraient rien à voir avec la psychopathologie mais seraient des mensonges ou des méprises; ou encore que les expériences exceptionnelles constituent des troubles psychiques non diagnostiqués dans la population générale [94-95].

D’une manière plus simpliste, l'évidence serait de dire qu'il n'y a pas un chevauchement complet: toutes les expériences exceptionnelles ne sont pas des troubles psychiques; et, réciproquement, tous les troubles psychiques ne sont pas des expériences exceptionnelles ayant fait l'objet d'un diagnostic psychiatrique. Si ce constat semble aller de soi, il existe néanmoins des discours extrémistes, qui ne proviennent pas toujours du seul public naïf, pour en affirmer le contraire. Ainsi, un discours psychopathologique à visée d'hygiène mentale a pu qualifier les expériences médiumniques de pathologiques en soi [96]; d'autres expériences exceptionnelles sont ensuite entrées dans la nosographie sans que ce soit toujours justifié par des études empiriques spécifiques [97]; des pionniers de la psychologie anomalistique ont ramené toutes les expériences exceptionnelles à des formes de psychopathologie liées aux mouvements ésotériques [73]; et un fantasme antipsychiatrique, actualisé notamment par la psychologie transpersonnelle [98], a pu être que, du fait d'un diagnostic différentiel biaisé, toutes les personnes témoignant d'une expérience exceptionnelle à un praticien de la santé mentale pouvaient être «enfermées » sur cette seule base [99]. D’un côté comme de l'autre, ces discours extrémistes révèlent des peurs : d'une part, la peur d'une montée de l'irrationnel mettant en péril les institutions ; et de l'autre, une peur du contrôle social exercé par les institutions laïques sur toutes les formes de spiritualité [71].

L'hypothèse du recouvrement complet entre expériences exceptionnelles et psychopathologie est peut-être la plus simpliste, mais c'est la plus largement partagée dans la population générale. Elle conduit fréquemment à contourner le circuit médico-psychologique classique pour éviter de se voir confirmer un diagnostic non souhaité. Ainsi, un grand nombre de personnes vivant des expériences exceptionnelles consultent des praticiens alternatifs (ce que nous pourrions appeler, à partir d'une 
expression de Tobie Nathan, du «paganisme thérapeutique » 4). Ce paganisme pourrait en retour alimenter la proportion des personnes vivant des expériences exceptionnelles qui échappent à tout diagnostic.

\subsection{Données en faveur d'un chevauchement partiel}

En l'absence de la démonstration d'un chevauchement complet entre expériences exceptionnelles et psychopathologie, des études plus fines ont pu évaluer l'hypothèse d'un chevauchement partiel, autrement dit de contributions de la psychopathologie aux expériences exceptionnelles et des expériences exceptionnelles à la psychopathologie (hypothèses $(b)$ et $(c)$ ). Ainsi, du fait de la prévalence des troubles psychiques d'une part et des expériences exceptionnelles d'autre part, une intersection entre les deux est inévitable.

Un des premiers endroits où cette intersection est clairement visible est dans la nosographie contemporaine basée sur le DSM-IV (Diagnostic and Statistical Manual of Mental disorders) et la CIM 10 (Classification Internationale des Maladies). Plusieurs troubles qui y sont décrits sont caractérisés par une liste de symptômes dans laquelle on retrouve plusieurs des phénomènes externes et internes liés aux expériences exceptionnelles (voir le tableau de Belz (19], p. 26).

L'hypothèse d'un chevauchement partiel est discutée à travers plusieurs voies de recherches ([19], pp. 20-25) :

A Expériences exceptionnelles et troubles dissociatifs. Les études actuelles montrent que la dissociation psychique est une fonction psychologique normale qui peut donner lieu à des conduites pathologiques. Cela réactualise la compréhension de la dissociation hystérique

4 Nathan utilise d'abord cette expression dans un plaidoyer pour le paganisme thérapeutique [100] ! Il en donnera plus tard une expression concrète : «cette espèce de polythéisme thérapeutique spontané de tous les patients du 
monde, qui n'hésitent jamais à enjamber les prétendues oppositions métaphysiques entre "naturel" et "surnaturel", entre "rationnel" et "irrationnel" et s'engagent successivement, parfois même concurremment, dans une démarche auprès d'un psychiatre, d'un psychothérapeute, mais aussi d'une voyante, d'un guérisseur, d'une église charismatique » ([101], p. 35)

qu'en avait Janet [102-103]. Des distinctions sont donc à faire, par exemple entre les personnes ayant des pratiques spirites impliquant des automatismes et les «psychoses médiumniques » [104]. Nous reviendrons sur cette voie par la suite.

A Expériences exceptionnelles et schizotypie. La schizotypie désigne une personnalité fortement susceptible de développer une schizophrénie, mais définie par des caractères propres, notamment le fait d'avoir des expériences «inhabituelles » [56]. Il y a des distinctions faites entre différentes formes de schizotypie, conduisant même parfois à prendre le concept comme une variable psychologique plus que comme une entité clinique [105]. Et certaines formes de schizotypies admettent qu'on puisse avoir des expériences exceptionnelles tout en ayant une bonne santé mentale [89-90, 87, 106].

A Expériences exceptionnelles et audition de voix. Il y a des situations, comme par exemple après avoir vécu une situation traumatique, où l'audition de voix peut être très fréquente (70 \% dans l'étude de Romme \& Escher [107]). Toute une littérature allemande et anglaise se développe, conjointement avec des associations de patients, pour dépathologiser l'entente de voix 5.

A Expériences exceptionnelles et désordres neuropsychologiques. Certaines expériences comme le déjà-vu [37], l'expérience de hors corps [108] ou de mort imminente [109] semblent avoir des racines neuropsychologiques. Elles sortiraient donc du champ de la psychopathologie stricto sensu.

A Expériences exceptionnelles et demande de conseil. Belz distingue un sous-groupe de ceux qui ont des expériences exceptionnelles et qui cherchent à être conseillés et aidés. L'IGPP a mené deux études diagnostiques, l'une rétrospective portant sur 844 cas [110], l'autre prospective sur 858 cas [25]. Elles montrent toutes deux que les cliniciens de l'IGPP considèrent qu'environ la moitié des gens qui viennent consulter présentent un symptôme de 
4 Evrard R, Le Maléfan P. Que changent les « entendeurs de voix » à l'écoute des hallucinations ? Article soumis.

A trouble psychique léger ou grave, alors que l'autre moitié n'aurait pas de signe clinique évident ([19], p. 25). Ces différences entre les proportions de diagnostic rendent donc essentielle la question du diagnostic différentiel.

\subsection{Chevauchement partiel et diagnostic différentiel}

Ces données issues de la recherche et de la clinique confirment l'enchevêtrement entre les expériences exceptionnelles et différentes catégories nosographiques. Cela conduit inévitablement à ce qu'une certaine phénoménologie puisse à la fois être décrite en termes de pathologies et d'expériences exceptionnelles. C'est un état de fait courant dans toute nosographie, et qui peut être amélioré à l'aide de critères de diagnostic différentiel qui permettent qu'un signe tombant ainsi dans plusieurs catégories ne corresponde finalement qu'à un nombre restreint de syndromes, afin que diagnostic et traitement soient appropriés.

Pour prendre un exemple concret, Corbeau [40] a sondé de nombreux professionnels de la santé aux Pays-Bas au sujet des expériences exceptionnelles. Pour les 41 professionnels qui ont eu un patient relatant une expérience de mort imminente, ces professionnels ont utilisé 9 catégories diagnostiques différentes du DSM-IV-R. Pour les 75 professionnels ayant reçu une personne témoignant d'un contact avec des esprits, 12 catégories diagnostiques distinctes furent sélectionnées, dont la mention d'un processus de deuil [18], d'un trouble psychotique non spécifié [9] ou aucun diagnostic [15]. Enfin, sur les 70 professionnels confrontés à une expérience de perception extra-sensorielle relatée par un patient, 17 catégories diagnostiques furent invoquées, dont $37 \%$ des diagnostics puisant dans les divers troubles de la personnalité et $11 \%$ de trouble psychotique non spécifié (pour une analyse, voir [41]).

Roberts [111] a montré à plusieurs reprises comment il était problématique de conclure quoi que ce 
soit à partir de ce chevauchement partiel entre expériences exceptionnelles et symptômes de troubles mentaux. La pose d'un diagnostic implique de prendre en compte une grande variété de critères, et pas seulement celui du type de vécu, en particulier quand il implique des jugements sur des « croyances ».

Il a pu être reproché à la nosographie contemporaine de n'avoir pas élaboré un diagnostic différentiel suffisant pour discriminer expériences exceptionnelles et troubles psychiques, et même d'être fondée en partie sur des amalgames et des généralisations abusives (par exemple, [112]). Des exemples historiques montrent par exemple l'emploi de démonstrations tautologiques au fondement de certaines entités cliniques, telle que la Psychose Hallucinatoire Chronique de Gilbert Ballet qui met inévitablement la transe médiumnique du côté de la psychose [113]. D’un autre côté, il n'a jamais été démontré que la phénoménologie en question était propre aux expériences exceptionnelles, même si des travaux progressent dans ce sens [33]. C'est justement parce que les mêmes phénomènes et expériences peuvent entrer dans la constitution ou la coloration de troubles tels que les délires qu'il y a une zone d'enchevêtrement.

Le postulat central de la clinique des expériences exceptionnelles est que ce chevauchement partiel est un fait, mais qu'une recherche plus approfondie peut permettre d'améliorer la qualité du diagnostic différentiel, c'est-à-dire d'ouvrir une palette plus large de diagnostics - plus légers ou plus graves - qui conduiront à des pratiques cliniques plus appropriées. Cette recherche peut impliquer le bouleversement de certaines entités cliniques qui se révéleraient trop ambiguës ou inapplicables. Elle ne vise pourtant pas à une dépathologisation générale des expériences exceptionnelles passant par un rejet de l'approche psychopathologique, car ce serait un parti pris contraire à ce constat d'un chevauchement partiel.

\section{Dissociation, traumatismes et expériences exceptionnelles}

Une autre hypothèse (hypothèse $(d)$ ) consiste à examiner si des variables tierces ne contribuent pas 
à la fois à l'expérience exceptionnelle et à la psychopathologie. Nous ne choisirons que deux exemples ayant fait l'objet d'un nombre conséquent de travaux : la dissociation et le traumatisme. Cette partie de la revue de la littérature impliquera de préciser davantage la distinction - longtemps négligée - entre croyances au paranormal et expériences exceptionnelles.

\subsection{Les expériences dissociatives}

Les expériences exceptionnelles et la dissociation ont une longue histoire commune [114]. Une définition reconnue de la dissociation dit qu'il s'agit «d'une séparation structurée de processus mentaux (pensées, émotions, conations, souvenirs et identités) qui sont généralement intégrés » [115]. L'ampleur de cette définition n'est pas sans poser problème : elle recouvre nombre de phénomènes décrits par des auteurs aussi différents que Myers, Freud, Janet, Jung ou encore Festinger. Or, dans une large mesure, notre compréhension de la relation entre dissociation et expériences exceptionnelles dépend de notre compréhension de la dissociation elle-même.

L'échelle des expériences dissociatives (Dissociative Experiences Scale, DES ; [116]) est largement utilisée pour mesurer la dissociation. Or, plusieurs études font état de davantage de revendications d'expériences exceptionnelles chez les personnes ayant des scores élevés à la DES [117-119; 45]. L'étude de Ross \& Joshi [45] conclut en fait une série d'études qui ont toutes mises en évidence davantage de traumatismes dans l'enfance et d'autres symptômes de dissociation chez les personnes vivant des expériences exceptionnelles. Parmi celles-ci, celles qui avaient été abusées sexuellement ou physiquement durant l'enfance relataient près de deux fois plus d'expériences que les autres $(2,3$ expériences exceptionnelles en moyenne chez 63 sujets abusés contre 1,2 en moyenne pour les 439 sujets non abusés).

La dissociation pourrait par ailleurs se manifester sous la forme d'expériences rencontrées fréquemment dans une population saine. Ainsi, 38,7\% de la population aurait au moins une fois dans sa vie une hallucination hypnagogique s'apparentant à la vision d'un fantôme ou à d'autres 
impressions étranges [120]; et $25 \%$ de la population vivrait, au moins une fois au cours de la vie, une paralysie du sommeil [121]. Le mécanisme neuropsychologique de cette expérience commence à être bien connue, et il est à même de rendre compte des récits d'«intruders », c'est-à-dire l'expérience hallucinatoire de la manifestation d'une présence non humaine dans la chambre à coucher [122-123]. Ce motif était autrefois représenté sous la forme de démons (incubes et succubes, cf. [124]), mais les motifs changeraient selon la culture, si bien que les extraterrestres en seraient une forme moderne (voir par exemple, [125]).

Plus largement, les états modifiés (ou «étranges », cf. [126]) de conscience se situent dans le même territoire à la croisée des chemins. Faisant l'objet d'un rejet et d'une fascination dans les sociétés occidentales, ils sont fréquemment associés à la psychopathologie alors que, dans d'autres cultures, ils sont ritualisés pour leurs vertus thérapeutiques (par exemple, [57]). Des recherches s'étendant sur plus d'un siècle montrent que ces états modifiés favorisent la survenue d'expériences paranormales [127-129]. Les vécus d'expériences paranormales médiatisées par les états modifiés de conscience seraient très fréquents dans la population générale et pourraient constituer des mécanismes d'adaptation ou de défense normaux pour le psychisme [130].

Les expériences dissociatives demeurent malgré tout à la lisière de la psychologie classique. Rares sont les cliniciens qui s'ouvrent officiellement à ces expériences alors même qu'ils les rencontrent dans leurs pratiques (par exemple, pour l'expérience de hors corps en clinique adulte et adolescente, [131-133]). La marginalisation de ces connaissances conduit par conséquent à un écart entre le besoin, exprimé par ceux qui les vivent, de mieux comprendre ces expériences et de savoir y faire face, et l'information fiable et adaptée qui leur est proposée [134]. Un rôle d'information, par le biais de la diffusion d'un «bagage commun » de connaissances sur les expériences exceptionnelles, serait un moyen de prévenir leurs conséquences négatives.

\subsection{Traumatismes et expériences exceptionnelles}


Une donnée importante pour la compréhension des expériences exceptionnelles et leur clinique est leur fréquente association avec des traumatismes antérieurs. Plusieurs études apportent un soutien conceptuel et empirique à cette question. Tout d'abord, nous tenons à préciser la distinction cruciale entre croyances au paranormal et expériences exceptionnelles.

\subsubsection{Croyances au paranormal et expériences exceptionnelles}

Dans la discussion qui va suivre, il est très important de différencier la notion de «croyances paranormales » et celle d' «expériences exceptionnelles». La littérature sur les croyances au paranormal est très abondante (pour une synthèse : [39]). Tandis que celle sur les expériences exceptionnelles est plus réduite. Par ailleurs, les deux phénomènes ne se mesurent pas de la même façon et n'auraient ni la même prévalence, ni les mêmes implications psychologiques.

Par exemple, Lawrence et Peters [135] ont fait passer des questionnaires à des membres de la Society for Psychical Research britannique (qui fut la première des sociétés savantes consacrées à la parapsychologie) pour évaluer leurs capacités de raisonnement. Ils ont trouvé que ceux qui avaient une forte croyance au paranormal montraient davantage de biais de raisonnement dans des tâches de déduction logique que ceux qui avaient vécu une expérience exceptionnelle. Toutefois, malgré quelques recouvrements, il n'y avait pas d'équivalence entre l'expérience et la croyance paranormales. Dans cette étude, $42,6 \%$ de ceux qui n'avaient qu'une faible expérience directe du paranormal affirmaient néanmoins croire fortement à sa réalité ; et, inversement, $11,1 \%$ des individus qui avaient une forte expérience du paranormal restaient sceptiques.

Autre exemple, Rattet et Bursik [136] ont fait passer des questionnaires de personnalité à des étudiants, en plus de questionnaires sur les croyances au paranormal et sur leurs éventuelles expériences de précognition. Alors qu'il s'agissait d'une population non-clinique, 65 des 107 étudiants $(60,7 \%)$ affirmèrent avoir vécu un type d'expérience précognitive. Les résultats montrèrent évidemment que la plupart de ces mêmes étudiants avaient des scores élevés de 
croyances au paranormal. Néanmoins, au niveau des caractéristiques de personnalité, des différences pouvaient être faites suivant qu'on regardait le groupe des «croyants » ou celui des « expérienceurs ». Le chevauchement entre les deux groupes n'empêchait pas la mise en évidence de distinctions claires dans leurs traits de personnalité. Les auteurs concluaient que les études antérieures qui avaient associé la paranormalité aux maladies mentales seraient peut-être à revoir en fonction de cette distinction entre croyance et expérience. Eux-mêmes imaginaient différents scénarios alternatifs reliant système de croyance paranormale et expérience, où soit la croyance est première, soit c'est l'expérience dont l'incongruence va entraîner une tentative de régulation.

Schriever [137] a renforcé empiriquement et conceptuellement la multidimensionalité des croyances au paranormal et aussi des non-croyances au paranormal. Les soi-disant «croyants » ne croient pas à tout et de la même façon; les soi-disant «sceptiques » ne rejettent pas tout sans discrimination. De plus, les croyants et les incroyants utiliseraient les mêmes types de raisonnement pour appuyer ou justifier leurs convictions.

Pechey et Halligan [138] ont sondé 1000 britanniques par téléphone. Ils ont observé que $89 \%$ de ceux qui disaient n'avoir pas eu d'expériences exceptionnelles croyaient tout de même modérément ou fortement à au moins une croyance paranormale. En somme, sur 1000 sondés, il n'y avait que 28 personnes n'endossant ni expérience exceptionnelle ni croyance paranormale. Leurs données montraient aussi l'inverse : $56 \%$ des personnes affirmant n'avoir aucune croyance paranormale reconnaissaient tout de même avoir vécu au moins une expérience exceptionnelle. Le rapport entre la croyance et l'expérience est donc loin d'être causal et unilatéral.

Malgré leur prévalence très élevée, on ne sait donc pas exactement comment l'un précède l'autre, ou par quoi croyances et expériences sont médiatisées. C'est dans cette recherche d'une explication du développement de croyances ou d'expériences paranormales que la question du traumatisme a pris une place importante. 


\subsubsection{Traumatismes antérieurs à des expériences exceptionnelles}

On peut remonter à Wilson \& Barber [139] pour une première recherche sur les traumas à l'enfance et les expériences exceptionnelles. Dans leur étude avec un petit échantillon, un plus grand nombre de traumas à l'enfance était mesuré chez les personnes «enclines à l'imaginaire », ce qui fut confirmé dans d'autres études [45].

Dans une perspective plus développementale, Irwin [140-141] a mis en évidence que les personnes ayant subi des traumatismes dans l'enfance, en particulier des abus sexuels ou des maltraitances par des parents alcooliques, relataient plus d'expériences exceptionnelles une fois adultes. C'est à partir de ces données qu'il construira un modèle dit «des facteurs dans l'enfance »: les expériences paranormales seraient liées avec l'inclination à l'imaginaire et avec un besoin important d'avoir un contrôle interpersonnel 6. Ce besoin de contrôle interpersonnel est associé à l'anxiété et à ce qui est perçu comme un manque de contrôle durant l'enfance, ce que Watt, Watson et Wilson [142] ont pu corréler au développement de croyances paranormales.

Ce modèle sera examiné par Lawrence avec d'autres données lui permettant d'apporter des modifications au modèle d'Irwin [143]. Il a confirmé que les expériences paranormales étaient corrélées avec les traumatismes et également avec une inclination excessive à l'imaginaire durant l'enfance (en anglais, childhood fantasy) 7. Additionnant les corrélations obtenues, son nouveau modèle proposait que « le traumatisme cause une tendance excessive à l'imaginaire qui cause des expériences qui causent une croyance, avec un lien direct du traumatisme à l'expérience » ([145], p. 213). Selon ce modèle, il y a donc à la fois une voie directe et une voie indirecte du traumatisme à l'expérience exceptionnelle, et les croyances paranormales sont plus souvent une conséquence de cette expérience que leur cause.

Plusieurs autres travaux confirment ce lien entre traumatismes à l'enfance et expériences exceptionnelles $[22,145,130]$ en venant tout de même questionner la part des traumatismes au long 
${ }^{6}$ Le contrôle interpersonnel ou social se définit ici comme le résultat de l'ensemble des possibilités d'agir efficacement vis-à-vis d'autrui.

${ }^{7}$ D'autres auteurs ont suggéré que le traumatisme «ouvrait » les individus à des états dissociatifs, préparant ainsi le terrain à des expériences exceptionnelles [144, 45].

cours, c'est-à-dire des situations traumatogènes ou des événements de vie négatifs, par rapport à celle des chocs traumatiques portés par un petit nombre d'événements. Perkins et Allen [145] ont ainsi pu mettre en évidence des différences dans les croyances paranormales selon le type de traumatismes. Ainsi, les violences physiques étaient associées à davantage de croyances au paranormal, par l'intermédiaire d'un plus grand sentiment de vulnérabilité, comme le suggèrent les auteurs. De la même façon, il y a aussi des différences entre le type de croyances au paranormal : les croyances religieuses traditionnelles semblent apporter plus de «sens » et de sécurité que la philosophie New Age (pour une discussion, voir [39]).

D'autres travaux sur la population générale ont mis en évidence un lien entre certaines mesures du traumatisme (cognitions négatives et toutes les variables de la dissociation) et la prédisposition à la fois aux hallucinations visuelles et auditives [146] . En analysant plus précisément ce lien, les auteurs ont montré que certains événements de vie en particulier (deuil, violence physique et abus émotionnel) prédisposaient aux hallucinations auditives, tandis que d'autres (dont le harcèlement) prédisposaient plutôt aux hallucinations visuelles. Cette étude conforte l'idée que le lien entre traumatismes et expériences exceptionnelles impliquerait de considérer des expériences traumatiques prises dans un sens assez large, mais aussi que ces expériences pourraient être comprises comme des solutions psychiques non psychotiques à certaines difficultés [92].

Au final, il y a eu encore assez peu d'études sur le lien entre traumatisme et expérience exceptionnelle mais toutes celles effectuées semblent aller dans le même sens, en suggérant que les deux sont liés. Au point que Ross et Joshi ont conclu :

«Les expériences paranormales et extrasensorielles sont communes dans la population générale. Elles peuvent représenter un aspect de la dissociation normale, ou une partie de la réaction dissociative à un traumatisme chronique durant l'enfance : en tant que tels, elles permettent de discriminer le groupe des individus fortement traumatisés des individus sans 
histoires d'abus durant l'enfance. Le paranormal, considéré comme un ensemble d'expériences subjectives, devrait être un objet de recherches sérieuses impliquant la psychiatrie et la psychologie académiques. » ([45], p. 361)

\section{Conclusion}

Nos revues de littérature pointent la nécessité d'une clinique différentielle des expériences exceptionnelles dont le rapport à la psychopathologie est complexe. Il n'y a pas une catégorie psychopathologique qui puisse s'appliquer uniformément du fait de la diversité des expériences exceptionnelles et des situations dans lesquelles elles se produisent. Ainsi, les catégories liées à la dissociation et au traumatisme possèdent des liens ambivalents avec les expériences exceptionnelles 8. Dans la littérature, ces vécus sont régulièrement associés à ces catégories sans se réduire à en être de strictes expressions. Il n'en découle pas encore de modèles parvenant à rendre compte de la dimensionnalité des expériences exceptionnelles et de leurs trajectoires parfois psychopathologiques. De nombreuses pistes restent donc à explorer dans ces domaines récemment structurés que sont la psychologie anomalistique et la clinique des expériences exceptionnelles.

Conflits d'intérêt: Pas de conflits d'intérêt. 
8 Les mêmes constats se font par rapport aux liens entre expériences exceptionnelles et les modes de fonctionnement psychique spécifiques de l'adolescence [147-148]. Voir également Evrard R, Rabeyron T. Psychose pour tous. La jeunesse au risque du « syndrome de psychose atténuée »? (Article soumis).

\section{Références}

1 Dean SR. Metapsychiatry and the ultraconscious. Am J Psychiatr $1971 ; 128$ : 154-55.

2 Neppe VM. Culture, Psychopathology and Psi: A Clinical Relationship. Parapsychological J of South Africa, 3(1) : 1-5.

3 Ullman M. Psychopathology and Psi Phenomena. In : Woman BB, editor. Handbook of Parapsychology. Van Nostrand Reinhold Company ; 1977. p. 557-573.

4 Calvesi A. The analytic relationship and its therapeutic factors from a parapsychological viewpoint. Psychoanal Rev 1983 ; 70(3) : 387-402.

5 Mathijsen F. Empirical research and paranormal beliefs: Going beyond the epistemological debate in favour of the individual. Arch Psycholo Religion 2009; 31(3): 1-15.

6 Irwin HJ, Watt C. An Introduction to Parapsychology. $5^{\text {th }}$ ed. Jefferson, NC: McFarland ; 2007.

7 Le Maléfan P. Discours psychiatrique et discours parapsychologique. Essai sur un historique et une actualité de leurs interactions. [Mémoire de D.E.A. d'Anthropologie et d'Ecologie Humaine]. Paris: Université Paris V ; 1986.

8 Chaperot C. Phénomènes d'allure télépathique dans la relation avec des patients schizophrènes : hypothèse d'une potentielle nocivité de la pensée soignante. Evol Psychiatr 2011; 76(2): 273 86.

9 Palmer G, Braud W. Exceptional Human Experiences, disclosure, and a more inclusive view of physical, psychological, and spiritual well-being. J Transpersonal Psychol 2002; 34(1): 29-61.

10 Maslow AH. Lessons from the peak-experiences. J Humanistic Psychol 1962; 2: 9-18.

11 Otto H. Multiple strength perception method, Minerva experience and others. In Otto H, editor. Explorations in human potentialities. Springfield, IL: Charles C. Thomas; 1966. p. 471-486.

12 Masters R. Consciousness and extraordinary phenomena. In : White J, editor. Psychic 
exploration: A challenge for science. New York: G. P. Putman’s Sons; 1974. p. 598-614.

13 Neher A. The psychology of transcendence. Englewood Cliffs, NJ: Prentice-Hall; 1980.

14 Helminiak DA. Neurology, psychology, and extraordinary religious experiences. J Religion Health 1984; 23: 33-46.

15 Nelson PL. Personality factors in the frequency of reported spontaneous praeternatural experiences. J Transpersonal Psychol 1989; 21: 193-210.

16 Murphy M. Explorations into the further evolution of human nature. New York : Jeremy P. Tarcher / Putnam Books; 1992.

17 McClenon J. Wondrous events: Foundations of religious beliefs. Philadelphia: University of Pennsylvania Press; 1994.

18 Van Dusen W. Beauty, wonder, and the mystical mind. West Chester, PA: Chrysalis Books; 1999.

19 Belz M. Außergewöhnliche Erfahrungen. Göttingen: Hogrefe ; 2009.

20 Fangmeier R. Entwicklung eines Konzepts für telefonische Beratung im Bereich der Beratung für Menschen mit Außergewöhnlichen Erfahrungen. [Thèse de psychologie non publiée] Freiburg: Université de Freiburg; 1999.

21 Gebhardt C. Brief und E-Mail als Medium von Beratung und Therapie: Analyse der Briefe und E-Mails von ratsuchenden Menschen mit Außergewöhnlichen Erfahrungen. [Thèse de psychologie non publiée] Feiburg; Université de Freiburg; 2000.

22 Spitz H. Emotionsregulation bei außergewöhnlichen Erfahrungen. Eine Fallstudie über Ratsuchende mit außergewöhnlichen Erfahrungen. [Mémoire de Master 2 Psychologie]. Freiburg; Albert-Ludwigs-Universität, Freiburg ; 2005.

23 Schäfer CS. Außergewöhnliche Erfahrungen: Konstruktion von Identität und Veränderung in autobiographischen Erzählungen. [Thèse de psychologie, Université Albert-Ludwigs de Freiburg] publiée en ligne : http://www.freidok.uni-freiburg.de/volltexte/6153/; 2008

24 Fach W. Phenomenological Aspects of Complementarity and Entanglement in Exceptional Human Experiences (ExE). Axiomathes $2011 ; 21(2)$ : 233-47.

25 Belz-Merk M, Fach W. Beratung und Hilfe für Menschen mit Außergewöhnlichen Erfahrungen. 
Psychotherapie. Psychosom Med Psychol 2005; 55: 256-65.

26 Le Maléfan P. Folie et spiritisme Histoire du discours psychopathologique sur la pratique du spiritisme, ses abords et ses avatars. 1850-1950. Paris : L'Harmattan ; 1999.

27 Evrard R. Les expériences réputées psychotiques dans la population générale : essai de problématisation. Ann Med Psychol 2011; 169(5): 282-7.

28 Cardeña E, Lynn SJ, Krippner S., editors. Varieties of anomalous experiences: Examining the Scientific Evidence. Washington, DC: American Psychological Association; 2000.

29 Lalande A. Vocabulaire technique et critique de la philosophie. Paris : Felix Alcan ; 1938.

30 Canguilhem G. Le Normal et le Pathologique. Paris : PUF; 1966.

31 Foucault M. Les anormaux. Cours au Collège de France, 1974-1975. Paris : Gallimard/Seuil ; 1999.

32 Castel R. La gestion des risques. De l'anti-psychiatrie à l'après-psychanalyse (1981). Paris: Editions de Minuit; 2011.

33 Rabeyron T, Chouvier B, Le Maléfan P. Clinique des expériences exceptionnelles : du trauma à la solution paranormale. Evol Psychiatr 2010; 75(4): 633-53.

34 Greeley AM. The sociology of the paranormal: A reconnaissance. Beverly Hills, CA: Sage ; 1975.

35 Greeley AM. The paranormal is normal: A sociologist looks at parapsychology. J Am Society for Psychical Res 1991; 85: 367-74.

36 Schmied-Knittel I, Schetsche M. Psi-Report Deutschland Eine repräsentative Bevölkerungsumfrag zu aussergewöhnlichen Erfahrungen. In : Bauer E, Schetsche M, editors. Alltägliche Wunder. Erfahrungen mit dem Übersinnlichen - wissenschaftliche Befunde. Würzburg: Ergon ; 2003.p. 13-38.

37 Neppe VM. Anomalous experience and psychopathology. In: Shapin B, Coly L, editors. Spontaneous Psi, Depth Psychology and Parapsychology. New York: Parapsychology Foundation; 1992. p. 163-180. 
38 Kennedy JE, Kanthamani H. An exploratory study of the effects of paranormal and spiritual experience on peoples' lives and well-being. J Am Soc Psychical Res 1995 ; 89 : 249-64.

39 Irwin HJ. The Psychology of Paranormal Belief: A Researcher's Handbook. Herfordshire: University of Hertfordshire Press ; 2009.

40 Corbeau I. Psi in de geestelijke gezondheidszorg. Tijdschrift voor Parapsychol 2004 ; $71(2)$ : 1015.

41 Eybrechts MV, Gerding JLF. Explorations in Clinical Parapsychology. In :Kramer W, Bauer E, Hövelmann G, editors. Perspectives of clinical parapsychology. Utrecht: HJBF Publications; 2012. p. 35-48.

42 Sannwald G. On the psychology of spontaneous paranormal phenomena. Int J Parapsychology 1963; 5: 274-292.

43 Bainbridge WS. Chariots of the gullible. The Skeptical Inquirer 1987 ; 3(2): 33-48.

44 Schmied-Knittel I, Schetsche M. Wie gewöhnlich ist das 'Aussergewöhnliche'? Eine wissenssoziologische Schlussbetrachtung. In Bauer E, Schetsche M, editors. Alltägliche Wunder. Erfahrungen mit dem Übersinnlichen - wissenschaftliche Befunde. Würzburg: Ergon ; 2003: 171-88.

45 Ross CA, Joshi S. Paranormal Experiences in the General Population. J Nerv Ment Dis 1992; 180(6): 357-61.

46 Hufford DJ. Commentary - Paranormal Experiences in the General Population. J Nerv Ment Dis 1992; 180(6): 362-68.

47 Bentall RP. Research into psychotic symptoms: are there implications for parapsychologists? Euro J Parapsych $2000 ; 15$ : 79-88.

48 Malinak DP, Hoyt MF, Patterson V. Adults' reactions to the death of a parent: A preliminary study. Am J Psychiatr $1979 ; 136$ : 1152-6.

49 Haraldsson E. Popular psychology, belief in life after death and reincarnation in the Nordic countries, Western and Eastern Europe. Nordic Psychology 2006, 58(2):171-80. 
50 Yamamoto J, Okonogi K, Iwasaki T, Yosimura S. Mourning in Japan. Am J Psychiatr 1969 ; $125: 1660-5$.

51 LaGrand L. Messages \& Miracles - Extraordinary Experiences of the bereaved. New York: Llewellyn; 2001.

52 Muhammad Gadit AA. Insightful hallucination: psychopathology or paranormal phenomenon. BMJ Case Reports, doi:10.1136/bcr.10.201; 2011.

53Allouch J. Érotique du deuil au temps de la mort sèche. 3ème éd. Paris : E.P.E.L ; 2011.

54 Le Maléfan P, Lemercier D. Clinique lacanienne du «fantôme » chez un adolescent en deuil. Evol Psychiatr 2012. http://dx.doi.org/10.1016/j.evopsy.2012.08.011

55 Hanus M Deuils normaux, deuils difficiles, deuils compliqués et deuils pathologiques. Ann Méd Psychol 2006; 164: 349-56.

56 Evrard, R. Schizotypie ou névrose ? la place des expériences réputées psychotiques. Pers psychiatr $2010 ; 49(3), 230-40$.

57 Mancini S. Postface. In: De Martino E. Le monde magique. Paris: Institut Synthelabo ; 1999, p. $285-584$.

58 Laplantine F, Aubrée M. La table, le livre et les esprits. Paris : J.-C Lattès ; 1990.

59 Méheust B. En soucoupes volantes - vers une ethnologie des récits d'enlèvements. Paris : Mercure de France ; 1985.

60 Weber M. L'Ethique protestante et l'esprit du capitalisme. Paris : Plon ; 1904-1905/1965.

61 Hansen, G. The Trickster and the Paranormal. Philadelphia: Xlibris books ; 2001.

62 Meheust, B. Somnambulisme et médiumnité (2 tomes). Paris: Les Empêcheurs de Penser en Rond ; 1999.

63 Hyman R. The Elusive Quarry: A Scientific Appraisal of Psychical Research. Buffalo, NY: Prometheus Books ; 1991.

64 Alcock JA, Burns J, Freeman A. Psi Wars - Getting to grips with the paranormal. Exeter: Imprint Academic ; 2003. 
65 Wiseman R, Watt C, editors. Parapsychology. Aldershot: Ashgate ; 2005.

66 Hergovich A. Der Glaube an Psi. Die Psychologie paranormaler Überzeugungen. Bern : Huber ; 2001.

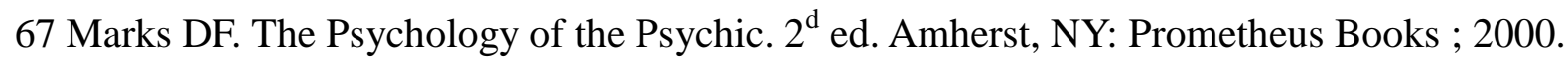

68 Shermer M. Why People Believe Weird Things: Pseudoscience, Superstition, and Other Confusions of Our Time. New York: Holt Paperbacks ; 2002.

69 Wooffitt R. Telling tales of the Unexpected: The Organization of Factual Discourse. Hertfordshire: Harvester Wheatsheaf; 1992.

70 Evrard R. Spirituality as an anti-psychopathological discourse on exceptional experiences. In Stark CA, Bonner DC, editors. Handbook on Spirituality: Belief Systems, Societal Impact and Roles in Coping. New York : Nova Science Publishers, 2012. p. 279-289.

71 Schetsche M. Soziale Kontrolle durch Pathologisierung? Konstruktion und Dekonstruktion, außergewöhnlicher Erfahrungen' in der Psychologie. In: Menzel B, Ratzke K, editors. Grenzenlose Konstruktivität? Standortbestimmung und Zukunftsperspektiven konstruktivistischer Theorien abweichenden Verhaltens . Opladen : Leske/Budrich ; 2003, p. 141-160.

72 Reed G. The psychology of anomalous experience. London: Hutchinson University Library ; 1972.

73 Zusne L, Jones WH, editors. Anomalistic psychology: A study of magical thinking. 2d ed. Hillsdale, NJ: Lawrene and Erlbaum; 1989.

74 Gilovich T. How we know what isn’t so. New York: Free Press; 1993.

75 Schick T, Vaughn L. How to think about weird things. Mountain View, CA: Mayfield; 1995.

76 French CC. Why I study anomalistic psychology. The Psychologist 2001; 14(7): 356-57.

77 Holt N, Simmonds-Moore C, Luke D, French C. Anomalistic psychology. London : Palgrave Macmillan ; 2012.

78 Roe CA, Sherwood S. Anomalistic Psychology. London: Palgrave Macmillan; 2011. 
79 Roe CA. Anomalistic psychology. In Holt N, Lewis R, editors. A2 Psychology 2009 AQA A Specification: The Student's Textbook. London: Crown House Publishing; 2009. p. 426-443.

80 Kramer WH, Bauer E, Hövelmann GH, editors. Clinical Aspects of Exeptional Human Experience. An Introductional Reader. Utrecht : HGBF ; 2012.

81 Simmonds-Moore C, editor. Exceptional Experience and Health. Essays on Mind, Body and Human Potential. North Carolina: McFarland ; 2012.

82 Murray C, editor. Mental Health and Anomalous Experiences. New York: Nova Science Publishers ; 2012.

83 Berenbaum H, Kerns J, Raghavan C. Anomalous experiences, peculiarity, and psychopathology. In : Cardeña E, Lynn SJ, Krippner S, editors. Varieties of Anomalous Experience: Examining the scientific evidence. Washington, DC: American Psychological Association; 2000, p. 2546.

84 Shafer MG. Self-actualization, mysticism, and psychic experience. [Thèse de psychologie non publiée]. Irvine: University of California; 1982.

85 Windholz G, Diamant L. Some personality traits of believers in extraordinary phenomena. Bull Psychonomic Society 1974; 3: 125-6.

86 Reinsel R. Dissociation and mental health in mediums and sensitives: a pilot survey. Proceedings of Presented Papers of Paarapsychological Association 2003 Annual Convention; 2003, p. 200-221.

87 McCreery C, Claridge G. Healthy schizotypy: the case of out-of-the-body experiences. Pers Ind Diff 2002; 32(1): 141-54.

88 Jackson M. Benign schizotypy? The case of spiritual experience. In: Claridge G, editor. Schizotypy: Implications for illness and health. New York: Oxford University Press; 1997, p. $227-250$.

89 Goulding A. Schizotypy models in relation to subjective health and paranormal beliefs and experiences. Pers Ind Diff 2004 ; 37(1) : 157-67. 
90 Goulding A. Healthy schizotypy in a population of paranormal believers and experients. Pers Ind Diff 2005; 38: 1069-83.

91 Schumaker JF. Mental health, belief deficit compensation, and paranormal beliefs. J Psychol $1987 ; 121(5): 451-7$.

92 Rabeyron T. Psychopathological and psychodynamic approaches to anomalous experiences: The concept of a paranormal solution. In : Murray CD, editor. Mental Health and Anomalous Experience. London: Nova Publishers ; 2012, p. 125-40.

93 Jacobi F, Hoyer J, Wittchen H-U. Seelische Gesundheit in Ost und West: Analysen auf der Grundlage des Bundesgesundheitssurveys. Zeitschrift für Klinische Psychologie und Psychotherapie $2004 ; 33(4): 251-60$.

94 Stefanis N, Hanssen M, Smirnis N, Avramopoulos D, Evdokimidis I, Stefanis C, Verdoux H, Van Os J. Evidence that three dimensions of psychosis have a distribution in the general population. Psychol Med $2002 ; 32(2): 347-58$.

95 Coelho C, Tierney I, Lamont P. Contacts by Distressed Individuals to UK Parapsychology and Anomalous Experience Academic Research Units - A Retrospective Survey Looking to the Future. Euro J Parapsychol 2008 ;23 : 31-59.

96 Burlet P. Du spiritisme considéré comme cause d'aliénation mentale. Lyon : Richard; 1863.

97 Neppe VM. Clinical psychiatry, psychopharmacology and anomalous experience. In: Coly L, McMahon JDS, editors. Psi and Clinical Practice. New York: Parapsychology Foundation; 1993, p. $145-162$.

98 Allix S, Bernstein P. Manuel clinique des expériences extraordinaires. Paris : InterEditions ; 2009.

99 Evrard R. Il mito dello schizofrenico con doti telepatiche. Quaderni di Parapsicologia 2011 ; $43(1): 35-43$.

100 Nathan T. Plaidoyer pour un paganisme thérapeutique. Psychol Médicale, $1988 ; 20$ : (pages inconnues). 
101 Nathan T. Nous ne sommes pas seuls au monde. Paris : Seuil/Les Empêcheurs de Penser en Rond ; 2001.

102 Janet P. L'état mental des hystériques, 2 tomes. Paris : Alcan; 1893-1894.

103 Garrabé J. La taxinomie actuelle des troubles dissociatifs. Evol Psychiatr 1999; 64: 717-26.

104 Bender H. Mediumistische Psychosen. Ein Beitrag zur Pathologie spiritistischer Praktiken. Z Parapsych Grenzgebiete Psychol 1958-1959; 2:173-201.

105 Holt N, Simmonds-Moore C, Moore S. Benign schizotypy: Investigating differences between clusters of schizotype on paranormal belief, creativity, intelligence and mental health. Proceedings of Presented papers of the 51st annual convention of the Parapsychological Association. Durham, North Carolina, USA: Parapsychological Association 2008, p. 82-96. Available from: http://documents.clubexpress.com/documents.ashx?key=epq8bOLeFHAnmb7wzGWcs2OE3 QnFXi60kzKvAKI\%2Blz/5VSEUQENyzhjT8D958PjR1i1J885Nbp93txOzTO09kw\%3D\%3 $\underline{\mathrm{D}}$

106 Schofield K, Claridge G. Paranormal experiences and mental health: Schizotypy as an underlying factor. Pers Ind Diff 2007; 43(7): 1908-16.

107 Romme M, Escher S. Hearing voices. Schizophr Bull, 1989; 15(2): 209-16.

108 Brugger P. Ich seh etwas, was du nicht siehst. Psychol Heute 2006; 9: 38-43.

109 Blanke O, Dieguez S. Leaving body and life behind: out-of-body and near-death experience. In Laureys S, Tononi G, editors. The Neurology of Consciousness: Cognitive Neuroscience and Neuropathology. Amsterdam : Elsevier ; 2009, p. 303-325.

110 Hofmann L, Wiedemer A. Ein Dokumentationssystem für aussergewöhnliche Erfahrungen (DAE). Z Parapsychol und Grenzgebiete der Psychol 1997 ; 39 :147-82.

111 Roberts G. Delusional belief systems and meaning in life: A preferred reality? British J Psychiatr $1991 ; 159$ (Suppl 14) : 19-28.

112 Grof S, Grof C, editors. Spiritual Emergency: When Personal Transformation Becomes a Crisis. 
Los Angeles : J.P Tarcher ; 1991.

113 Le Maléfan P. Gilbert Ballet et la médiumnité. Contribution à l'histoire de la PHC et des divisions subjectives. Evol Psychiatr 2003 ; 68 : 63-72.

114 Alvarado CS. Dissociation in Britain during the late nineteenth century: The Society for Psychical Research, 1882-1900. J Trauma Diss 2002; 3: 9-33.

115 Spiegel D, Cardeña E. Disintegrated experience: The dissociative disorders revisited. J Ab Psychol, $1991 ; 100: 366-78$.

116 Bernstein EM, Putnam FW. Development, reliability, and validity of a dissociation scale. J Nerv Ment Dis $1986 ; 174$ : 727-735.

117 Richards DG. A study of the correlations between subjective psychic experiences and dissociative experiences. Dissociation 1991; 4: 83-91.

118 Zingrone N, Alvarado CS. Psychic and dissocative experiences: A preliminary report. The Parapsychological Association. $37^{\text {th }}$ Annual Convention: Proceedings of Presented Papers. Durham, North Carolina, USA: Parapsychological Association ; 1994, p. 489-501.

119 Wolfradt U. Dissociative experiences, trait anxiety and paranormal beliefs. Pers Ind Diff 1997; 23(1): 15-9.

120 Ohayon MM. Prevalence of hallucinations and their pathological associations in the general population. Psychiatr Res 2000; 97(2-3): 153-64.

121 Cheyne JA. Sleep paralysis episode frequency and number, types, and structure of Associated Hallucinations. J Sleep Res 2005 ; 14 : 319-24.

122 Cheyne JA. The ominous numinous: Sensed presence and 'other' hallucinations. J Consciousness Studies $2001 ; 8: 133-50$.

123 Cheyne JA, Rueffer SD, Newby-Clark IR. Hypnagogic and hypnopompic hallucinations during sleep paralysis: Neurological and cultural construction of the night-mare. Consciousness Cognition 1999; 8: 319-37.

124 Garçon M, Vinchon J. Le Diable. Étude historique, critique et médicale. Paris : Gallimard ; 
1926.

125 French CC, Santomauro J, Hamilton V, Fox R, Thalbourne MA. Psychological aspects of the alien contact experience. Cortex 2008 ; 44(10) : 1387-95.

126 Valla JP. Les états étranges de la conscience. Paris : PUF ; 1992.

127 Myers FWH. La personnalité humaine (1903). Paris : Exergue ; 2011.

128 Tart CT. Altered States of Consciousness. A Book of Readings. New York: John Wiley; 1969.

129 Cardeña E, Winkelman M, editors. Altering Consciousness (2 vol.). Santa Barbara, CA: Praeger Publishers; 2011.

130 Rabeyron T, Watt C. Exploring the relationship between paranormal experiences, mental health and mental boundaries, and psi. Pers Ind Diff 2010; 48(3): 487-92.

131 Roisin J. La sortie du corps et autres expériences extrêmes du corps en situations de traumatisme. Revue francophone du Stress et du Trauma 2009 ; 9(2) : 71-80.

132 Le Maléfan P. La «sortie hors du corps » est-elle pensable par nos modèles cliniques et psychopathologiques? Essai de clinique d'une marge. A propos d'un cas. Evol Psychiatr 2005 ; $70: 513-34$

133 Le Maléfan P. La «sortie hors du corps » comme nouveau tropisme pour la clinique du corps ? Recherches en Psychanalyse $2011 ; 11$ : 38-46.

134 Milton J. Effects of "paranormal” experiences on people's lives: An unusual survey of spontaneous cases. J Society for Psychical Res 1992; 58: 314-23.

135 Lawrence E, Peters E. Reasoning in Believers in the Paranormal. J Nerv Ment Dis 2004 ; 192 : 727-33.

136 Rattet SL, Bursik K. Investigating the personality correlates of paranormal belief and precognitive experiences. Pers Ind Diff $2001 ; 31: 433-44$.

137 Schriever F. Grenzbereiche der Realitätserfassung. Ein Erklärungsmodell auf der Basis individueller Lebenserfahrungen. Berlin: Retriever ; 1998.

138 Pechey R, Halligan P. Prevalence and correlates of anomalous experiences in a large non- 
clinical sample. Psychology and Psychotherapy: Theory, Res Practice 2012; 85(2): 150-62.

139 Wilson SC, Barber TX. The fantasy-prone personality: Implications for understanding imagery, hypnosis, and parapsychological phenomena. In : Sheikh AA, editor. Imagery: Current theory, research, and application. New York : Wiley; 1983. p. 340-390.

140 Irwin HJ. Origins and functions of paranormal belief: the role of childhood trauma and interpersonal control. J Am Society for Psychical Res 1992 ; 86(3) : 199-208.

141 Irwin HJ. Childhood trauma and the origins of paranormal belief: a constructive replication. Psychol Reports 1994 ; 74(1) : 107-11.

142 Watt C, Watson S, Wilson L. Cognitive and psychological mediators of anxiety: Evidence from a study of paranormal belief and perceived childhood control. Pers Ind Diff 2007; 42(2): 33543.

143 Lawrence TR, Edwards C, Barraclough N, Church S, Hetherington F. Modelling childhood causes of paranormal belief and experience: Childhood trauma and childhood fantasy. Personality and Individual Differences 1995, 19(2) : 209-15.

144 Ring K. Projet Omega. Expérences du troisième type - N.D.E.. Paris : Editions du Rocher; 1994

145 Perkins SL, Allen R. Childhood physical abuse and differential development of paranormal belief systems. J Nerv Ment Dis 2006 ; 194(5) : 349-55.

146 Morrison AP, Petersen T. Trauma, Metacognition And Predisposition To Hallucinations In NonPatients. Behav Cogn Psychother 2003; 31(03): 235-46.

147 Le Maléfan P. «La vérité est ailleurs » : La place du paranormal à l'adolescence comme mode de traitement du réel pubertaire. Adolescence 2008 ; 26(3): 709-21.

148 Evrard R. Psychiser le Maître absolu : solutions pubertaires par le paranormal. Adolescence $2010 ; 28(4): 841-54$. 